\title{
Dentist morale falling alongside dwindling income
}

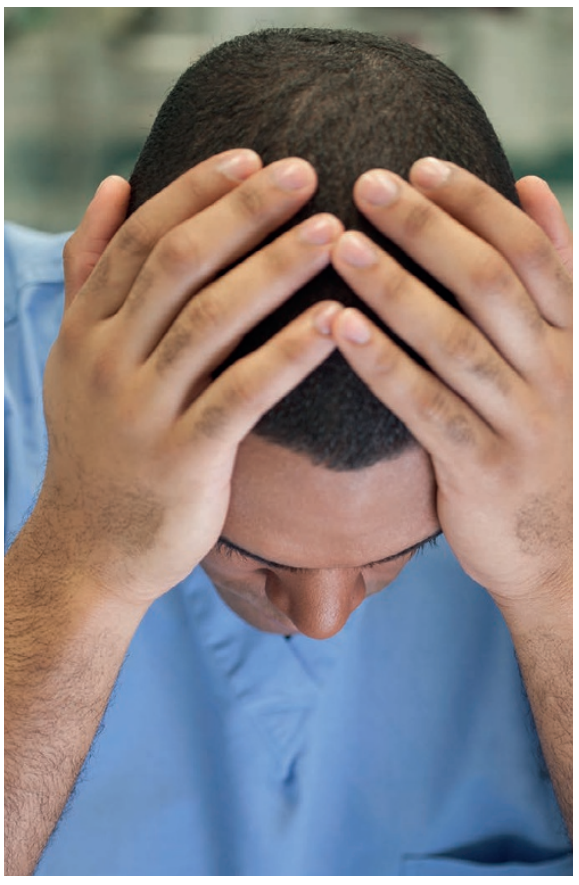

Morale of general dental practitioners has fallen steadily over the past decade at the same time as declining income levels, according to official data released by NHS Digital.

The official figures paint a worrying picture of a profession losing confidence, earning less and struggling to meet the needs of patients with less time spent on clinical work - a situation described by the BDA as a system 'running on fumes'.

The data ${ }^{1}$ from NHS Digital published on 30 August 2018 showed that NHS dentists in England and Wales had experienced a 35\% pay squeeze over the last decade.

Real incomes for practice-owning dentists fell by as much as $£ 47,000$, and their associates by more than $£ 23,000$ over the last decade.

In 2016-17, there was a $0.7 \%$ drop in taxable income of self-employed dentists (Providing-Performer and Performer Only dentists) in England and Wales from $£ 69,200$ in 2015-16 to $£ 68,700$ in 2016-17.

In 2016-17, there was a $4.3 \%$ decrease in taxable income of self-employed dentists (Principal and Associate dentists) in Northern Ireland, from $£ 69,400$ in 2015-16 to $£ 66,400$ in 2016-17.

However, in Scotland, 2016-17 saw a 0.1\% increase in taxable income of self-employed dentists from $£ 67,700$ in 2015 - 16 to $£ 67,800$ in 2016-17.
Other data ${ }^{2}$ on dental working hours, working patterns, motivation and morale published at the same time showed that morale has fallen to lowest levels since 2000 .

Morale described as 'high' or 'very high' in 2016-17 was only found in a fifth (20.1\%) of principals and a quarter (24.9\%) of associates.

In contrast, morale recorded as 'low' or 'very low' was $56 \%$ for principals and $48.1 \%$ for associates.

Growing disillusionment with the job was evident as the figures showed that almost two thirds (62.7\%) of principal dentists in England and Wales said they often thought about leaving general dental practice in 2017-18 compared to $57.2 \%$ in 2015-16.

More than half (56.1\%) of associate dentists said they often thought of leaving in 2017-18 compared to $47.6 \%$ in 2015-16.

A similar pattern was evident in Scotland where $69.3 \%$ of principal dentists said they often thought of leaving in 2017-18 compared to $57.1 \%$ in $2015-16$ while $57.1 \%$ of associate dentists considered leaving in 2017-18 compared to $45.9 \%$ in 2015-16. Northern Ireland's dentists had similar opinions.

The time spent on clinical work rather than administration or management has also been reducing as the data showed that in 2017-18, dentists in England and Wales spent, on average, $77.2 \%$ of their time on clinical work - a decrease from $85.4 \%$ in 2008-09. Similar trends were apparent in Scotland and Northern Ireland.

BDA Chair of General Dental Practice Henrik Overgaard-Nielsen said: 'Austerity is meant to be over, but across the UK NHS dentistry is running on fumes. We've seen a drop in real incomes without precedent in the public sector. The results are predictable, morale at an all-time low, recruitment and retention problems mounting, as patients wait longer or travel further for care.'

NHS Digital. Dental Working Hours 2016/17 and 2017/18: Working Patterns, Motivation and Morale [PAS]. 30 August 2018. https://digital.nhs.uk/data-and-information/ publications/statistical/dental-working-hours/2016-17and-2017-18-working-patterns-motivation-and-morale (accessed on 3 September 2018).

2. NHS Digital. Dental Earnings and Expenses Estimates 2016/17. 30 August 2018. https://digital.nhs.uk/ data-and-information/publications/statistical/dental-earnings-and-expenses-estimates/2016-17 (accessed 3 September 2018).

\section{Dental care may benefit patients set for cancer surgery}

Patients due to undergo cancer surgery who receive good preoperative oral care from a dentist appear to be significantly less likely to develop postoperative complications, suggests a recent study ${ }^{1}$ published in the British Journal of Surgery on 8 August 2018.

Japanese researchers found that the involvement of dentists in the preoperative management of patients who are undergoing cancer surgery may be essential for decreasing postoperative complications such as pneumonia or even death.

Improving patients' oral hygiene is an option for preventing postoperative pneumonia that may be caused by aspiration of oral and pharyngeal secretions.

Several previous studies have suggested that preoperative oral care might be associated with a decrease in postoperative pneumonia and mortality after cardiac or cancer surgery, but it remains unclear because these studies were limited by small sample sizes and a small number of participating institutions.

Therefore a team of researchers from the University of Tokyo carried out a retrospective cohort study to assess the association between preoperative oral care and postoperative complications among patients due to have major cancer surgery.

Using data from the National Database of Health Insurance Claims in Japan - an all-patient administrative claims database developed by Japan's Ministry of Health, Labour and Welfare that covers more than 126 million people - they studied 509,179 patients who underwent surgery for head and neck, oesophageal, gastric, colorectal, lung or liver cancer between May 2012 and December 2015.

Patients were monitored 12 months before surgery until the day of 
4 discharge from hospital or the end of the study (31 March 2016).

Of these patients, $16 \%$ received preoperative oral care from a dentist. When a surgeon requested that a dentist provide preoperative oral care to a patient with cancer, the dentist checked the patient's oral condition, provided professional tooth cleaning, taught the patient self-cleaning methods for the teeth, and provided any treatment needed.

Analysis of the data showed that 15,724 patients (3.09\%) developed postoperative pneumonia and $1,734(0.34 \%)$ died within 30 days of surgery.

After adjustments, preoperative oral care by a dentist was linked with a decrease in postoperative pneumonia from $3.76 \%$ to
$3.28 \%$ and death within 30 days from $0.42 \%$ to $0.30 \%$.

The authors said: 'The findings could help improve strategies for the prevention of postoperative complications.'

1. Ishimaru M, Matsui $H$, Ono S, Hagiwara $Y$, Morita $\mathrm{K}$, Yasunaga $\mathrm{H}$. Preoperative oral care and effect on postoperative complications after major cancer surgery. Br J Surg 2018; DOI:10.1002/bjs.10915. (accessed on 14 August 2018).

\section{Largest oral HPV study in England shows lower than expected infection rates}

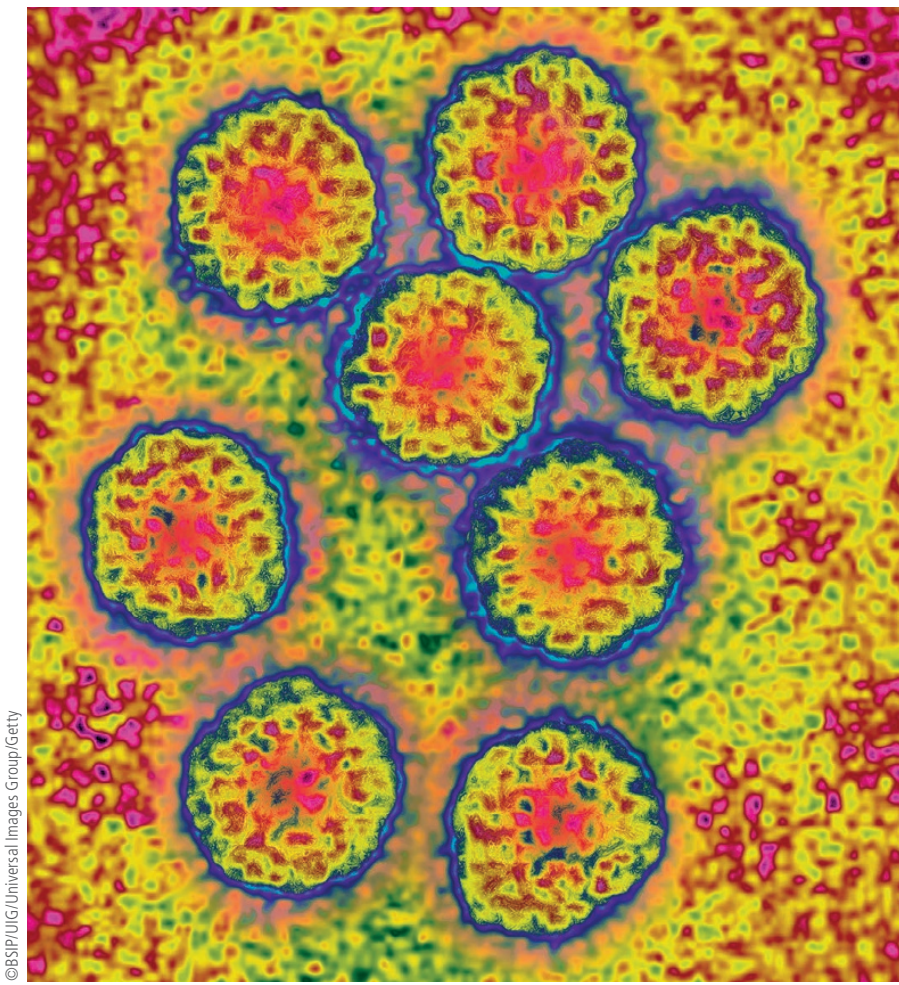

The largest study so far of high risk human papillomavirus (HR-HPV) oral infection in England has shown that infection rates are lower than expected, compared to previous studies.

The research, ${ }^{1}$ conducted by the University of Sheffield, also showed that smoking and sexual behaviour were risk factors for oral HPV infection, which can lead to oropharyngeal (throat) cancer.

The study was published in the online journal BMJ Open on 19 August 2018 and was led by the University's Professor Hilary Powers, Dr Vanessa Hearnden and Dr Craig Murdoch and funded by the World Cancer Research Fund UK.

It coincides with the announcement of a new UK HPV vaccine programme for boys which will reduce the risk of HR-HPV related cancers.

Rates of oropharyngeal cancer (OPC) are rising worldwide, attributable to an increase in the incidence of oral infection with HR-HPV.

For the new study - described as the largest of its kind in England - researchers recruited 700 men and women in Sheffield and looked for HR-HPV infection and also asked participants lifestyle questions relating to their sexual history and tobacco use over the period of April 2013 to August 2014.

Analysis of the results showed that $2.2 \%$ of people were infected with oral HR-HPV infection with 0.7\% positive for HPV 16 or HPV 18.

There are large variations in oral HR-HPV prevalence globally, but this study showed lower rates compared to previous US ${ }^{2}$ and Scottish ${ }^{3}$ studies which both found $3.7 \%$ of individuals positive for oral HR-HPV.

Former smokers were significantly more likely to be HR-HPV positive compared with those that had never smoked and the study also found that participants with a greater number of sexual or oral sexual partners were more likely to be HR-HPV positive.

Dr Vanessa Hearnden, from the Department of Materials Science and Engineering at the University of Sheffield, said: 'Previous studies have been US-focused or in smaller UK studies in London or Scotland. This is the first study in the North of England and found lower rates of oral high-risk human papillomavirus infection.

'We fully support the newly announced HPV vaccination programme for boys which will reduce the risk of HPV-related cancers including throat cancer in men and will also provide further prevention of cervical cancers through herd immunity.

'However, we found the majority of individuals testing positive for high risk strains of HPV were actually positive for strains other than those covered by the current vaccine (HPV 16 and HPV 18). This shows the need to consider newer vaccines which protect against more HPV strains in the future and for individuals to be aware of lifestyle risk factors such as number of sexual partners and tobacco use.'

Dr Craig Murdoch, from the University's School of Clinical Dentistry, said: 'Many people associate the HPV virus with cervical cancer but there is less recognition of the fact that HPV causes oropharyngeal cancer, and unfortunately, the prevalence of this cancer has increased dramatically in the past few years.'

1. Hearnden V, Murdoch C, D'Apice K, Duthie S, Hayward N, and Powers H J. Oral human papillomavirus infection in England and associated risk factors: a case-control study. BM Open 2018; e022497. DOI:10.1136/bmjopen-2018-022497. http://dx.doi.org/10.1136/ bmjopen-2018-022497 (accessed on 20 August 2018).

2. Gillison M L, Broutian T, Pickard R K et al. Prevalence of oral HPV infection in the United States, 2009-2010. JAMA 2012; 307: 693-703. https://jamanetwork.com/journals/jama/ fullarticle/1104983. (accessed on 20 August 2018).

3. Conway D I, Robertson C, Gray H et al. Human Papilloma Virus (HPV) Oral Prevalence in Scotland (HOPSCOTCH): a feasibility study in dental settings, PLOS One 2016; 11: e0165847. http://journals.plos.org/plosone/article?id=10.1371/journal.pone.0165847. (accessed on 20 August 2018). 